\title{
Reivindicaciones del derecho a una educación propia: La figura del educador comunitario
} Claiming the right of differential education:
the role of the community teacher

Investigación

\author{
Maike Kreisel \\ Universidad Veracruzana - México \\ maike_k@yahoo.de
}

Recibido: 31 de marzo de 2016 / Aceptado: 30 de junio de 2016

\section{Resumen}

Este trabajo aborda algunos hallazgos de un estudio etnográfico realizado en el modelo educativo alternativo de las Secundarias Comunitarias Indígenas en el contexto comunitario del estado de Oaxaca, México. Se centra en la relevancia de la figura docente para mediar la práctica y los procesos de apropiación de una propuesta que aboga por la reivindicación del derecho de las comunidades originarias a la construcción de sus propios sistemas educativos. El modelo cuestiona las formas clásicas de la educación escolarizada, además de los procesos históricos de asimilación, aculturación y exclusión de los pueblos indígenas a través de las políticas educativas del Estado-nación.

A través de proyectos de investigación, el modelo busca partir de los conocimientos comunitarios y de la participación de los actores locales para reivindicar las epistemes indígenas y generar procesos educativos situados y pertinentes, en los que se fomenta el diálogo con los saberes de las ciencias y con otros espacios culturales. Los educadores comunitarios se convierten en intermediarios y promotores de una educación diferencial que implica replantear su propia identidad étnica, profesional y sus saberes pedagógicos, y los lleva a posicionarse como actores etnopolíticos en defensa del derecho a una educación propia de los pueblos originarios.

Palabras clave: educación indígena, derecho a la educación, interculturalidad, epistemología, participación de la comunidad

\begin{abstract}
This paper reports some of the findings of an ethnographic study about the educational practices of the Secondary Community Schools as an alternative school project in the indigenous context of Oaxaca, Mexico. It focuses on the relevance of the role adopted by teachers as they mediate differential learning processes and promote the local appropriation of the project. The school project claims the Indigenous Peoples' right to create their own educational systems, and questions traditional school forms as well as the processes of historic acculturation, assimilation and exclusion of the indigenous peoples through governmental education policy.

Through the implementation of research projects as learning strategies, the project pretends to foster the recognition of indigenous epistemologies and to create relevant and situated learning processes, promoting the participation of local actors and the dialogue with their knowledges, as well as the exploration of science and different cultural spaces. The project's teachers become intermediaries and agents of a differential education proposal, being confronted with the need of redefining their own ethnic and professional identities. Furthermore, they get to take position as ethnopolitical actors in defense of Indigenous Peoples' educational rights.
\end{abstract}

Keywords: indigenous education, educational rights, interculturality, epistemology, community participation.

Forma sugerida de citar: Kreisel, Maike (2016). Reivindicaciones del derecho a una educación propia: la figura del educador comunitario. Alteridad, 11(1), pp. 21-32. 


\section{Introducción}

El presente artículo resume algunos de los hallazgos de un estudio etnográfico de larga duración que buscó conocer cómo se resignifica la institución escolar en la práctica educativa del modelo educativo alternativo de las Secundarias Comunitarias Indígenas de Oaxaca, estado sureño de México en el que el $65.73 \%$ de la población se considera indígena (INEGI, 2015). Este proyecto se enmarca en el contexto de las propuestas educativas con carácter etnopolítico que han emergido en las últimas décadas en el contexto latinoamericano y que abogan por la reivindicación de las culturas originarias y afroamericanas, frente a los procesos de aculturación y asimilación cultural históricamente fomentados por los Estados-nación.

Estas propuestas demandan el reconocimiento al derecho de los pueblos a una educación propia y pertinente, asentado en la legislación local y acuerdos internacionales (González Apodaca y Rojas, 2013). En el caso de México, la Ley General de Educación, la Ley General de los Derechos Lingüísticos de los Pueblos Indígenas (2003), pero también el reciente Programa Especial de Educación Intercultural 2014-2018 (2013), entre otros, reconocen dichos derechos; a ello se suman acuerdos internacionales como la Declaración Universal de los Derechos de los Pueblos Indígenas de las Naciones Unidas (2007) y el Convenio 169 de la Organización Internacional del Trabajo (1989).

Las Secundarias Comunitarias Indígenas de Oaxaca se constituyen como un modelo diferencial de educación secundaria y atienden un nivel educativo que el subsistema de educación indígena en México no cubre. Reconocidas por el Instituto Estatal de Educación Pública de Oaxaca, comenzaron a trabajar desde el año 2004 en diferentes regiones del estado; actualmente, cuentan con diez escuelas. Su diseño curricular y metodológico se distingue por proponer una aproximación diferencial a la educación escolarizada; en lugar de las asignaturas, se trabaja a través de proyectos de investigación centrados en preocu- paciones comunitarias, para así situar los aprendizajes y volverlos significativos para los estudiantes y la comunidad. Se parte de la recuperación de los conocimientos comunitarios para dialogar con los contenidos considerados como científicos, pretendiendo reivindicar la epistemología indígena y trascender la fragmentación del conocimiento por disciplinas. Se impulsa la participación de los padres de familia y comuneros en los procesos de aprendizaje y en la toma de decisiones, fortaleciendo la vinculación entre escuela y comunidad.

En el presente artículo, pretendemos mostrar cómo, en este contexto, cobran importancia las resignificaciones que los docentes indígenas realizan del modelo, convirtiéndose en actores clave para la mediación de los procesos de apropiación local y la interacción entre escuela y comunidad. Queremos resaltar cómo los docentes se constituyen, además, en sujetos pro-activos y políticos en defensa del derecho a una educación adecuada a las necesidades de los pueblos. Esto conlleva procesos de reflexión y rearticulación de las identidades docentes, como profesionistas y actores comunitarios.

González Apodaca (2006), desde la experiencia oaxaqueña de los Bachilleratos Integrales Comunitarios, estudia el papel de intermediarios que los profesionistas y maestros asumen en estos procesos de apropiación de un modelo educativo diferencial. La autora analiza cómo los docentes "se posicionan en la arena de la escolarización intercultural regional como figuras de intermediación político-cultural" (p.4). En el estado de Oaxaca, la historia de la redefinición del sujeto docente como actor etnopolítico se acentúa en el marco del movimiento magisterial y el papel asumido por la Sección XXII como fracción local de peso político del Sindicato Nacional de los Trabajadores de la Educación (SNTE). Aunque no pretendemos, en el presente texto, abundar sobre la complejidad de estas constelaciones sindicalespolíticas, hacemos aquí breve referencia a ellas para contextualizar esta investigación.

Las mismas Secundarias Comunitarias de Oaxaca son de origen magisterial; fueron creadas como parte de las propuestas pedagógicas del Movimiento Pedagógico impulsado por la Coalición 
de Maestros y Promotores Indígenas de Oaxaca (CMPIO). La CMPIO, como Jefatura de Zonas de Supervisión 21-Plan Piloto, fue fundada desde 1974; en el año 1981 comenzó a participar como delegación en la Sección XXII, a partir de su involucramiento en el movimiento magisterial. Como actor local, sin embargo, se ha posicionado de manera crítica frente a algunas tendencias de este mismo movimiento, a veces enfocado más a las políticas laborales que a los quehaceres propiamente pedagógicos. Después de un análisis exhaustivo, la CMPIO se propuso iniciar nuevos procesos formativos, en respuesta a las necesidades de las comunidades originarias.

Desde el año 1995, la CMPIO comenzó a impulsar el así llamado Movimiento Pedagógico (MP), en cuyo seno han realizado diferentes iniciativas caracterizadas por su dimensión comunitaria y la innovación pedagógica. Meyer (2010) señala que el objetivo central del MP ha sido la formación de maestros de acuerdo a las necesidades de los estudiantes y las comunidades, para lo cual se ha buscado identificar las estructuras sociales, las historias y los valores locales "para hacer de ellos la base de su currículo, su pedagogía, y sus relaciones comunales" (p.86). El MP se concibió desde sus inicios como un proceso colectivo, con la participación de las comunidades pero también actores académicos externos.

Entre las iniciativas de la CMPIO, contamos la formación de maestros en aspectos de la comunalidad, pedagogía y lingüística, además de la etnografía áulica para la recuperación de la propia práctica; diplomados en educación inicial, además del proyecto del tequio pedagógico (Meyer, 2010) y los nidos de lengua (Meyer y Soberanes, 2009), y finalmente, también el diseño del proyecto de las mismas Secundarias Comunitarias Indígenas.

De la misma manera que el MP, la creación de las Secundarias Comunitarias se concibió como un proceso participativo.

Cuando digo que las secundarias son parte del movimiento pedagógico, que de ahí nacieron, estoy incluyendo a toda la gente que ha participado en estas cosas. Aunque no sean de CMPIO (...) Pero yo creo que la idea que predominó fue lo que se fue construyendo acá en un equi- po que se conformó de maestros de aquí de la jefatura y del Departamento de Educación Indígena, junto con [Javier Sánchez] Pereyra y con Luis Madrigal (E-FSB-CMPIO).

Este eje de la participación atraviesa la concepción del modelo desde la perspectiva de la comunalidad como propuesta de pensamiento filosófico-etnopolítico oaxaqueño (Rendón Monzón, 2003). El valor que la propuesta educativa atribuye a lo colectivo se basa en el pensamiento de las comunidades originarias; "la secundaria que se propone desde Oaxaca, pretende su inserción comunitaria plena, por tanto pretende también una nueva forma de educar y de gestión en y con la comunidad" (IEEPO/ESCI, 2007, p. 7). Maldonado (2002) argumenta que la comunalidad implica la "sensación de pertenencia" y "voluntad de ser parte de la comunidad" (p. 4). Para Guerrero y Massieu (2012), "se trata de una propuesta para nombrar la vida cotidiana de la gente en comunidad, y para posibilitar el florecimiento de ese modo de vida; o dicho técnicamente, de un planteamiento epistemológico y político fundado en lo propio" (p. 241).

La adscripción del modelo al pensamiento de la comunalidad implica determinadas formas pedagógicas, organizativas y de toma de decisiones, en las que se busca la vinculación con los actores y las prácticas comunitarias, contrario las distancias entre escuela y comunidad que han prevalecido históricamente. Los educadores de las secundarias han tenido que replantear sus saberes pedagógicos, ya que sus propias trayectorias escolares y formativas, en gran parte, fueron de corte tradicional. Se han apropiado de otras maneras de ser docente, resignificando la misma propuesta educativa en el proceso. Presentamos a continuación algunos de los referentes teóricos que nos han apoyado en el análisis.

\section{Identidad docente, saberes \\ pedagógicos y apropiaciones}

Bolívar (2006) comprende la identidad docente a partir de la idea de la trayectoria; lo que configura a esta identidad es la biografía del maestro, su currículum vitae (p. 25). En estas configuraciones 
influyen no únicamente la formación como profesionista, sino también la propia historia escolar, personal y familiar. Es a partir de estas historias y sus cruces que el docente se constituye como profesionista, con los conocimientos que requiere para la labor cotidiana.

Mercado (1991) señala que los saberes de los maestros se relacionan, generalmente, con prácticas concretas y que se construyen "desde la propia historia e intereses", combinándose conocimientos de diferentes momentos y ámbitos de la biografía docente (p. 60). Estas prácticas se enfocan a la solución de problemas y pasan por un proceso de apropiación del oficio de maestro. Una faceta relevante de la apropiación es su carácter colectivo; es "atravesado por varias dimensiones: la historia social en su cruce con la historia personal de cada maestro; las relaciones que se dan entre los maestros, con sus alumnos y con los diversos sujetos y sectores sociales del lugar de trabajo" (Mercado, 1991, p. 60).

En relación al carácter colectivo del proceso de apropiación de los saberes, retomamos, con Gasché (2005), el concepto de interaprendizaje, con el que el autor se refiere a la construcción de conocimiento en procesos formativos entre actores indígenas y no indígenas, que se basan en "una relación de reciprocidad (...) con el beneficio mutuo de la ampliación de la capacidad de acción políticamente trascendente" (p. 13). Nosotros consideramos, en relación a ello, los procesos tanto de la creación del modelo de las Secundarias Comunitarias, donde participaron actores de diferentes adscripciones étnicas, académicas e institucionales, como los de la formación continua de los educadores.

La co-construcción de conocimientos es un proceso dinámico que forma parte de la apropiación y resignificación de la misma propuesta educativa, pero también, a través de ello, de la escuela como institución. Se trasciende la dimensión áulica al considerar, para los procesos de aprendizaje, no únicamente la interacción entre estudiantes y docentes, sino que se parte, a través de procesos de investigación impulsados por los mismos jóvenes, de los saberes locales. Estos se recuperan a través de entrevistas a los "portadores del conocimiento comunitario", es decir, a ancianos y ancianas poseedores del conocimiento de la experiencia, además de otros actores comunitarios.

Este diálogo conlleva, como labor fomentada por los mismos educadores, el análisis de las asimetrías históricas de las relaciones entre conocimientos; de "este entramado de saberpoder" que Rivera Cusicanqui (2008) ha llamado "colonialismo interno" (p.6) y que implica la continuidad de relaciones coloniales, en nuestro caso, a través de instituciones del Estado como el mismo sistema de educación pública que perpetúa la jerarquización e imposición de saberes y formas de vida. En este sentido, el diálogo de saberes en las Secundarias Comunitarias implica volver a arraigar la educación "en el suelo seguro de las comunidades" (Rengifo, 2009, p. 12), fortaleciendo el enlace con las prácticas sociales locales y la experiencia comunitaria como fuente de saber. Además, se busca reconocer, a través de procesos de reflexión metacultural (Jiménez Naranjo, 2009), las lógicas culturales subyacentes a los saberes y las prácticas y poder valorar las consecuencias de unas y otras en relación a sus implicaciones para un posible "buen vivir" comunal (Vanhulst \& Beling, 2013).

Consideramos, en relación a ello, el proceso de aprendizaje como dialógico y situado, lo cual atañe también a la misma formación del educador, entendida como proceso permanente. Desde la perspectiva del saber como práctica y como experiencia, pero también como proceso colectivo, Lave y Wegner (1991) arguyen que "el aprendizaje es una dimensión integral e inseparable de la práctica social" (pp. 31), donde se evidencian el carácter relacional de todo conocimiento y aprendizaje, "el carácter negociado del significado" y la naturaleza comprometida del aprendizaje, dirigida a la solución de problemas (p. 33).

En el contexto de las secundarias comunitarias, el concepto del aprendizaje situado se vuelve relevante tanto para la formación de los estudiantes, como para la consolidación del modelo y sus resignificaciones por los actores implicados en la práctica. Este proceso conlleva la redefinición de 
la identidad profesional del educador, además de la reconceptualización de la escuela como institución, con miras a una educación comunitaria para los pueblos originarios.

La labor en un proyecto educativo diferencial implica procesos de apropiación pero también el manejo de conflictos y tensiones. Los coordinadores y asesores del modelo han fomentado el cuestionamiento consciente del papel tradicional de la escuela y del maestro, concebido desde lógicas más verticales. Se pretende fortalecer la identificación de los educadores con una pedagogía distinta a través de un proceso colectivo de formación, además de la recuperación de los saberes pedagógicos generados la práctica cotidiana y la reflexión de ésta. Las resignificaciones se relacionan con procesos de apropiación, los cuales, en nuestro contexto concreto, también evocan posicionamientos etnopolíticos.

González Apodaca (2014) comprende las propuestas educativas comunitarias e interculturales con carácter etnopolítico como aquellas que "se caracterizan por la incorporación de un eje político transversal a sus dimensiones lingüísticas, culturales, pedagógicas, curriculares, administrativas y de gestión", y donde el carácter etnopolítico incide en modelar las prácticas de los actores en un campo de diferentes tensiones (p. 1145). De esta manera, en las reconfiguraciones de la identidad docente influyen, además de la formación y de la historia escolar, personal y familiar, también la pertenencia comunitaria y étnica, y la adscripción sindical e ideológica, un aspecto relevante en el contexto magisterial oaxaqueño.

González Apodaca (2010) considera que la organización de los maestros del estado de Oaxaca a través de determinadas estructuras sindicales conlleva la formación de "un habitus particular" que incide en la configuración de la vida y labor, pero también en las expresiones identitarias, culturales y lingüísticas de los maestros oaxaqueños (p. 233). En nuestro caso, la dimensión etnopolítica está presente en los discursos y las prácticas docentes, y también se refleja en cómo los educadores de las Secundarias Comunitarias promueven la apropiación del modelo por los actores locales. Retomamos aquí las aproximaciones teóricas propuestas por González Apodaca (2006) acerca de "las apropiaciones locales del proceso de escolarización como fenómeno multidimensional” (p. 35).

Estas articulaciones se encuentran mediadas por los procesos de formación docente, fomentados continuamente desde el modelo, además de los aprendizajes generados en la interacción cotidiana en los mismos centros escolares, entre pares, con los estudiantes y los integrantes de la comunidad, es decir, a través de la experiencia pedagógica y comunitaria. Nos basamos aquí en una concepción de las identidades como procesos socialmente construidos, de "carácter intersubjetivo y relacional” (Giménez, 1997, p. 12), pues esta construcción identitaria ocurre a través de procesos dialógicos, entre educadores, con los asesores, pero también en el enfrentamiento con las críticas y resistencias de algunos actores al modelo. Los educadores de las Secundarias Comunitarias elaboran la diferencia frente a la práctica y el discurso de otros, pues "la identidad de un actor social emerge y se afirma sólo en la confrontación con otras identidades en el proceso de interacción social" (Giménez, 1997, p. 12).

En cuanto a nuestro interés en la vinculación entre escuela y comunidad, González Apodaca (2010) profundiza que el involucramiento en propuestas educativas con enfoque comunitario conlleva para el docente "la necesidad de abandonar la posición generalmente distante y autosuficiente del maestro, y acercarse a los miembros de su comunidad" para poder lograr el pretendido diálogo cultural. Transita de considerarse como "conocedor a priori de la cultura", hacia una visión más plural de los actores que participan en los procesos formativos de los niños y jóvenes (p. 236).

\section{Enfoque metodológico}

La presente investigación se basó en el enfoque etnográfico, buscando recuperar, desde una mirada hermenéutica, cómo los actores significan su propia realidad social, sus "acciones, discursos e instituciones” (Velasco y Díaz de Rada, 
1997, p.104). Considerando la importancia de la inmersión en el mundo de vida de los actores (Rockwell, 2011), se optó por una prolongada estancia en campo, para lo cual la investigadora radicó el estado de Oaxaca de enero 2014 a febrero 2015. La investigación se planteó como estudio de caso, realizado en una de las diez comunidades en las que se insertan las Secundarias Comunitarias. Además, se participó en eventos de las Secundarias, reuniones y cursos de formación docente, en diferentes espacios. Como métodos, se optó por la observación participante, la entrevista etnográfica, semi-estructurada y abierta, y los grupos de discusión. Los principales instrumentos utilizados fueron el diario de campo, además de guías flexibles de entrevista. Para el análisis, se transcribieron las grabaciones de audio y se analizaron con apoyo del programa Atlas. ti, estableciendo categorías de análisis. Se procedió de manera inductiva, buscando el diálogo entre referentes empíricos y teóricos, y regresando oportunamente a campo. El informe final de esta investigación se encuentra actualmente en proceso de redacción; algunos hallazgos se presentan a continuación.

\section{Intermediaciones para la apropiación $y$ defensa de una educación propia}

En un proyecto alternativo como las Secundarias Comunitarias Indígenas, hemos de considerar diferentes factores que hacen del papel de los educadores un elemento clave para impulsar tanto la articulación e integración del modelo a nivel local, en interacción con padres de familia, comuneros, autoridades y estudiantes, como también para posicionarlo frente a las incidencias del contexto histórico, social y político más amplio. Como propuesta en muchos sentidos opuesta a las formas tradicionales de la institución escolar, la participación pro-activa de los educadores resulta central para dar a conocer los fundamentos y formas de trabajar, y en su caso, defenderlo con base en el derecho a una educación propia para los pueblos originarios. Se requiere, por ende, de un nivel importante de apropiación por parte de los docentes, que es mediado por procesos de resignificación constantes.

Para su labor educativa, los docentes han tenido que replantearse su quehacer profesional; el currículum del modelo, que se concibe como procesual y se encuentra en continua reestructuración a través del trabajo por proyectos, implica desarrollar diferentes formas pedagógicas y resignificar el papel del educador. Al respecto, uno de los docentes expresa que "lo que tiene este modelo, exige mucho trabajo del educador (...) enseñar de otra forma, es ahí donde ya exige mucho análisis, mucha reflexión, buscar bastante, observar bastante en la comunidad" (E-Conrado-educador).

Ser educador de las secundarias comunitarias implica para los docentes reafirmarse como profesionistas dispuestos a romper con las lógicas clásicas de la educación escolarizada y construir una práctica innovadora en búsqueda del diálogo comunitario. La adscripción a la educación comunitaria requiere de un compromiso personal y conlleva un proceso de reflexión y de de-construcción de significados socialmente adquiridos, a través de la propia historia escolar y la inmersión en el contexto de la sociedad más amplia, excluyente. Esta toma de conciencia y resignificación ha sido acompañada por los asesores de las Secundarias Comunitarias.

No es fácil de entender lo comunitario, es complicado, y pues tenemos una formación, estamos dentro de una sociedad donde hay posiciones, lo mismo lo podemos ver en la comunidad cuando dicen que la lengua, no, ya no va a servir para un niño, un estudiante. Desde ahí ya nacimos, dentro de esa visión, que en nuestra cultura no hay conocimiento, no hay ciencia. Entonces de ahí, la asesoría sí es la que nos ayuda también, tanto en la parte de reflexionar, aparte de lo metodológico (E-Conrado-educador).

De esta manera, los docentes también han tenido que posicionarse; su labor en las secundarias conlleva la confrontación permanente con la identidad institucional específica de la propuesta, 
que al ser distinta a las modalidades oficiales, como la telesecundaria o la secundaria técnica, también genera críticas en diferentes espacios. Observamos cómo los educadores han asumido su pertenencia a la propuesta.

Esto es lo que queremos nosotros, tanto como educadores y yo creo que también en las comunidades aún sienten eso, aunque haya críticas fuertes de que no es congruente con lo que estamos acostumbrados de tipos de escuelas formales o federalizadas, ¿no? Y por eso, sí, ya me apropié del modelo (E-Maximino-educador)

Los docentes, al asumirse como parte del modelo, se ven impulsados a reflexionar sobre su propia identidad y pertenencia a un pueblo originario, aunque las comunidades en las que laboran pocas veces coincidan con sus lugares de procedencia. Resulta relevante el que puedan trazar continuidades entre su contexto personal e identidad étnica y la tarea que han asumido como promotores activos de la educación comunitaria. Así, constata uno de ellos que "él se puede defender porque su papá le enseñó el proceso de la milpa y del café (...) y que la secundaria busca esta comunicación entre conocimientos" (Ob-conversación informal-Carlos-educador). En algunos educadores se observa cómo la concientización acerca de los procesos de discriminación vividos por los pueblos incide en las decisiones que toman para su vida personal, como la de volver a enseñar la lengua indígena a sus hijos.

Investigadora: ¿Tú te has llegado a sentir identificada con el modelo?

Educadora: Sí porque primero soy hablante de una lengua indígena. $Y$ eso también me da orgullo, yo no me avergüenzo de hablar una lengua indígena porque varias personas se avergüenzan y no, peor tantito, se salen a la ciudad, no quieren hablar (E-Elena-educadora).

Elena me contó que ya está hablando más en chinanteco con su niña y le dice que pronuncie las palabras, pero aún no pronuncia bien. Su esposo sigue hablándole en español, pero sí la idea es que aprenda las dos lenguas (Ob-conversación informal-escuela).
Para los educadores, resignificar su propia pertenencia a las comunidades originarias, identificarse con la filosofía de la comunalidad, pero también reconocerse como parte del colectivo de integrantes del modelo de las Secundarias Comunitarias, es parte del proceso de apropiación del proyecto. A través de procesos de interaprendizaje, en interlocución con asesores del modelo, pero también a través de la lectura y otras estrategias de autoformación, los educadores han construido un andamiaje intelectual en el que se apoyan. De esta manera, "se posicionan como intelectuales locales" que actúan como intermediarios del modelo "hacia fuera" y "hacia dentro" (González Apodaca, 2006, p.161). Buscan el permanente diálogo con los actores locales para impulsar la paulatina apropiación de la propuesta por la comunidad. Una figura escolar relevante es el Consejo Representativo de Padres de Familia (CR); es nombrado por la asamblea comunitaria y ha de participar activamente en la toma de decisiones e intervenir en la vinculación entre escuela y comunidad. Los educadores mantienen una comunicación constante con sus integrantes y fomentan su familiarización con el modelo.

Educador [al CR]: Muchas veces las comunidades ya no le dan importancia a la lengua, por ejemplo. Anteriormente, la escuela prohibía y castigaba a los que hablaban su lengua, el zapoteco, el chinanteco, el mixe, (...). Entonces por esta razón se fue perdiendo la cultura de las comunidades a partir de la misma escuela. (...) Por esa razón también surge esta secundaria porque tiene esta finalidad, fortalecer, recuperar la cultura (Ob-transcripción-reunión educadores/nuevo CR).

Se pretende generar reflexión y conciencia acerca del derecho de las culturas originarias a una educación propia que tome en cuenta el aprendizaje de las lenguas y los conocimientos indígenas y se enfoque a las necesidades locales. Las propuestas educativas que defienden tales derechos se posicionan frente a una situación compleja de tensiones de poder históricamente configuradas, que conllevan 
negociaciones permanentes a nivel comunitario, social e institucional. Estas confrontaciones implican elaborar distinciones de lo que se considera como "lo propio" y "lo ajeno"; requieren tomar un distanciamiento y conciencia de las prácticas culturales - aquellas consideradas como propias resultan etnificadas en el proceso, incidiendo en procesos identitarios (Dietz, 2013, pp. 182-183).

En la práctica educativa, se observa una tendencia a la dicotomización. En la investigación comunitaria, se impulsa la resignificación de "lo propio" en términos afirmativos y se promueve la reflexión crítica de los procesos de aculturación presentes en la comunidad. Esta tendencia lleva a la contrastación entre las prácticas culturales comunitarias y las incidencias de "lo ajeno", donde las últimas se perciben, en relación a los procesos de dominación cultural vividos, frecuentemente como negativas. Observamos, por ejemplo, que los estudiantes detectan la presencia de procesos de "contaminación cultural, lingüística y de pensamiento" en su comunidad (Ob-tercer grado).

El diálogo entre culturas que promueve la escuela incide en la reconfiguración de las fronteras étnicas desde la mirada de la reivindicación de los pueblos. De la Peña (2008) constata que la intermediación entre culturas no implica necesariamente que éstas "se conciban como compartimentos estancos; por el contrario, las fronteras culturales se van constituyendo en el propio proceso de intermediación" (p. 1). La reafirmación de la identidad étnica en la práctica escolar se relaciona con los posicionamientos etnopolíticos que sustentan el proyecto y se reflejan en la discursividad de sus actores; toca, a un nivel más amplio, el derecho colectivo a la autodeterminación de los pueblos indígenas.

La dimensión etnopolítica se relaciona además con la situación emergente de incertidumbre que las Secundarias Comunitarias -como propuesta alternativa que cuenta con el reconocimiento estatal, mas no federal- enfrentan ante la reforma del sistema educativo gestionada desde finales del año 2012. Ésta afecta al modelo y sus recursos a través de la recentralización administrativa (Olmeda, 2014), y es vista por diferentes actores académicos y sociales como de carácter neoliberal y de control educativo (López Aguilar, 2013). Los educadores perciben a estas tendencias como "un retroceso" para la educación indígena y las comunidades (Ob-aula-3er grado); participan en actividades sindicales en defensa del modelo y procuran informar e involucrar a padres de familia y autoridades en reuniones y eventos educativos. De esta manera, también los estudiantes participan y argumentan a favor de su escuela.

Estudiante: Podríamos decir que la secundaria comunitaria las ventajas que tiene es que los estudiantes expresan con sus compañeros, pueden expresar sus ideas, pueden problematizar, investigar (...) en cambio en las otras escuelas pues los estudiantes que hay allá sólo se ubican en contestar en páginas, en libros y en otras materias. En cambio, en esta secundaria, pues los estudiantes pueden expresar sus ideas. $Y$ las desventajas que tiene pues - ¡es que no tiene desventaja! (G-grupo de discusión 3er grado)

Los procesos de apropiación de la escuela, a nivel de los actores comunitarios, sin embargo, son complejos. Dietz (2013) enfatiza, con Jiménez Naranjo (2009), que no ha de partirse de la suposición de procesos necesariamente armónicos entre escuela y comunidad; se trata más bien de "procesos de apropiación, imposición, enajenación y autonomización" que también "generan divergencias de intereses tanto dentro de la comunidad como entre ésta y la institución escolar" (pp.182-183). Observamos que, en la práctica, los educadores participan continuamente de un proceso de negociación; afirman que en algunas comunidades, "la gente como que aún no logra visualizar muy bien lo que pretende el modelo (...). Están fuertemente en que se trabaje como secundarias técnicas, por asignaturas. Como ellos, digamos, han caído en el tradicionalismo, desde preescolar o desde primaria, (...) piensan que en la secundaria comunitaria no se aprende" (E-Elena-educadora).

Los docentes han analizado el contexto en el que se inserta el proyecto y están conscientes de las dificultades por las que puede transitar el egresado en su futuro camino, en un entorno en 
el que la escolarización se sigue perfilando como un medio excluyente, con contenidos ajenos a las realidades que viven los estudiantes indígenas:

También tiene que ver (...) la discriminación. Porque son de una comunidad, no tienen los medios ni siquiera, apenas sí logran cursar un bachillerato con los poquitos recursos que tienen, y todo el día están con temáticas [escolares] que tienen que ver con otra clase, ser un empresario, ¿no? Pero sí es mucha discriminación (E-Conrado).

Estas configuraciones del entorno mayor, aunado a los procesos de cambio a nivel comunitario, influyen necesariamente sobre las expectativas de las familias hacia la escuela. Las comunidades oaxaqueñas están pasando por transformaciones a través del contacto cultural, acentuado actualmente por los procesos de migración, la llegada de productos comerciales y de la tecnología, como la televisión, los celulares y las computadoras. Los educadores observan cómo estos cambios se reflejan en los estudiantes; "ya de pronto hablan algunas palabras, algunos conceptos y dice uno, esto lo más seguro lo vio en la tele, en alguna novela, en alguna película" (E-educador-Conrado).

González Apodaca (2006) plantea la desterritorialización de la identidad como un fenómeno que participa en las reconfiguraciones identitarias locales (p.149). En nuestro caso, podríamos aludir a la multiplicidad de espacios en contacto que inciden en la configuración de identidades igualmente plurales, situación que se acentuará en el caso de los jóvenes quienes ahora tienen acceso a las redes sociales en Internet y a otros espacios culturales a través de la televisión. Haesbaert (2013), más allá de la desterritorialización de las identidades, plantea la posibilidad de vivir entre diferentes culturas y "distintas identidades territoriales que se cruzan” (p. 38).

González Apodaca (2006) argumenta que es justamente parte de las transformaciones que viven las comunidades oaxaqueñas, los procesos migratorios, los avances tecnológicos, en coe- xistencia con las prácticas "premodernas" que se conservan en la agricultura local, que se "perfilan nuevas modalidades a la intermediación" del maestro indígena (p. 343). Creemos que los educadores de las Secundarias Comunitarias de Oaxaca se encuentran en un lugar que demanda la capacidad de moverse en distintos escenarios y percibir la multiplicidad de intereses e inquietudes que mueven a los estudiantes, sin excluir ninguna de estas dimensiones. El coordinador del modelo expresa que la formación de los jóvenes como futuros comuneros que se pretende es precisamente en la reflexividad de la vida comunitaria, en su contacto y diálogo con otros espacios:

Porque ya son gentes que piensan diferente, valoran lo suyo, valoran lo propio. $\mathrm{Y}$ entonces caminas en una autonomía ya propia. Pero ya no depende de nadie, porque ya reflexionas, ya piensas, ya sabes qué hacer con los recursos naturales, con la vida comunitaria. Si migras, ya sabes a qué vas a migrar. Pero sabes que vas a regresar. Si vas a liderear, sabes que vas a liderear y cómo vas a liderear en la comunidad. Ese es el papel de la educación comunitaria (E-Coordinador).

Es así como las Secundarias Comunitarias se constituyen como un actor importante en el panorama de las propuestas, negociaciones y resistencias presentes en el estado de Oaxaca en torno a la definición y concreción de la educación para las comunidades originarias. El modelo de las secundarias ha llegado a convertirse en emblemático para una diversidad de actores, pero sobre todo para el magisterio indígena. Representa una experiencia exitosa, distintiva por sus propias características, de lo que sería un modelo educativo propio con fundamentos teóricos, metodológicos, pero además, con casi doce años de experiencia pedagógica: "Es una forma muy diferente, única, una forma muy singular de hacer educación secundaria, y para nosotros digamos que es la punta de lanza de lo que se está haciendo en materia de educación indígena en Oaxaca" (E-funcionario Dirección de Educación Indígena). 


\section{Conclusiones}

¿De qué sirve tener el derecho a usar su propia lengua si no hay escuelas o medios de comunicación para ello? (Stavenhagen, 2010, p. 76)

En el presente artículo, nos hemos centrado en la relevancia que adquiere el papel del docente en una propuesta alternativa de educación secundaria que cuestiona los esquemas clásicos de la educación escolarizada, convirtiendo a la comunidad, sus conocimientos y necesidades en el centro de su quehacer educativo. Para desempeñar su labor en el modelo, los educadores de las Secundarias Comunitarias de Oaxaca se enfrentan con la necesidad de replantear su identidad profesional y de desarrollar nuevos saberes pedagógicos.

En los proyectos de investigación que estructuran las actividades educativas, se pretende generar el diálogo entre la cultura comunitaria, los conocimientos de las ciencias y otras culturas, fomentando la reivindicación de la epistemología indígena. De esta manera, la secundaria busca vincularse con las prácticas sociales y la experiencia comunitaria como fuente de aprendizaje. En la práctica educativa, este fortalecimiento de "lo propio" se enfrenta con las transformaciones paulatinas de las comunidades a través del contacto entre culturas; la migración y los medios, pero también la influencia de la misma educación escolarizada como proceso histórico de asimilación de los pueblos a la sociedad mayoritaria, han generado procesos de cambio cultural.

Estas constelaciones han requerido del educador la sensibilidad y capacidad de observación del contexto y de las necesidades que genera el contacto entre culturas. Éste implica tensiones y desplazamientos, pero también el derecho de las comunidades de elegir y acceder a diferentes formas de vida, en condiciones de igualdad. Los educadores comunitarios participan de la mediación inter-cultural en permanente interacción y relación de inter-aprendizaje con el contexto en el que laboran, con los comuneros, los padres de familia y estudiantes, pues desde la perspectiva del modelo, esta es la base para construir una educación pertinente y propia, basada en una nueva vinculación entre escuela y comunidad.

Como propuesta alternativa, el modelo ha estado expuesto a cuestionamientos en diferentes espacios. Los educadores han actuado como mediadores frente a una diversidad de actores en el contexto comunitario, magisterial y educativo, por la reivindicación del derecho a una educación propia. De esta manera, su labor adquiere una dimensión etnopolítica compleja, que atraviesa la discursividad y los quehaceres cotidianos, en la mediación de los procesos de apropiación y defensa. En ello inciden las tendencias de las políticas educativas a nivel nacional y los procesos reivindicativos y de resistencia más amplios de los pueblos originarios y del magisterio de Oaxaca, que no alcanzamos a profundizar aquí.

Este artículo pretende iluminar, no obstante, cómo esta propuesta de educación comunitaria indígena se convierte en un espacio de negociación y redefinición de la educación escolarizada, donde se resignifican las preocupaciones y los intereses de las mismas comunidades originarias en relación a la formación de los jóvenes, pero también se dialogan y reafirman las perspectivas para un "buen vivir" comunitario como opción para un futuro sostenible en una sociedad plural, basado en el reconocimiento de la diferencia y de las múltiples formas de vida. Los aprendizajes que los actores de la propuesta han adquirido a través de su experiencia en la práctica, acompañada de procesos de diálogo y reflexión co-construidos, se perfilan como una contribución importante para concebir alternativas educativas para los niños y jóvenes, más allá de lo local.

Frente a las tendencias de las políticas educativas verticales y de corte neoliberal, la experiencia de las Secundarias Comunitarias de Oaxaca nos permite vislumbrar la urgencia del diálogo intercultural para aportar a la construcción de propuestas educativas pertinentes, basadas en una nueva vinculación entre escuela y entorno, y que formen parte de las apuestas por una mayor justicia cognitiva y social (Santos, 2007). 


\section{Referencias Bibliográficas}

Bolívar, A. (2006). El currículum como curso de la vida y la formación del profesorado. Universitas Tarraconensis. Revista de Ciències de l'Educació, edición especial, 25-44.

Caffentzis, G. \& Federici, S. (2013). Commons against and beyond capitalism. Upping the Anti: a journal of theory and action (15), 83-91 Recuperado de: http://sduk.us/money/sg_ commons_againstbeyond_capitalism.pdf

De la Peña, G. (2002). La educación indígena. Consideraciones críticas. Sinéctica (20), 46-53. Recuperado de: http://sinectica.iteso. $\mathrm{mx} /$ assets/files/articulos/20_la_educacion_ indigena_consideraciones_criticas.pdf

(2008). La antropología social y cultural en México. Versión preliminar VIII/08. Ponencia magistral. Congreso de Antropología en España y en Europa. Recuperado de: http:// pendientedemigracion.ucm.es/info/antrosim/docs/DelapenaMexico.pdf

Dietz, G. (2013). Comunidad e interculturalidad entre lo propio y lo ajeno: hacia una gramática de la diversidad. En: Baronnet, B. \& Tapia, U. (coords.). Educación e interculturalidad: política y políticas (pp. 177-199). Cuernavaca: UNAMCRIM

Esteva, G. (2012). Regenerar el tejido social de la esperanza. Polis [En línea] (33). Recuperado de: https://polis.revues.org/8487

Gasché, J. (2005). Hacia una propuesta curricular intercultural en un mundo global. Revista Interamericana de Educación de Adultos, 27(1) 177-200. Recuperado de: http:/tumbi.crefal.edu.mx/rieda/ images/rieda-2005-1/contrapunto3.pdf

Giménez, G. (1997). Materiales para una teoría de las identidades sociales. Revista Frontera Norte, 9(18). México: COLEF. Recuperado de: http://www.colef.mx/fronteranorte/articulos/FN18/1-f18_Materiales_para_una_ teor\%EDa_de_las_identidades_sociales.pdf

González Apodaca, E. (2006). Etnicidad, intermediación y escuela: tres iniciativas de bachillerato intercultural en la región mixe. Tesis doctoral. México: UAM Iztapalapa.

(2010). Una experiencia de apropiación de la pedagogía inductiva intercultural entre maestros y formadores de docentes en Oaxaca, México. Cuadernos Interculturales, 8(14), 227-243 (2014). Identidad étno-comunitaria y experiencias escolares de egresados de un bachillerato comunitario Ayuujk. RMIE, 19(63), 1141-1173. Recuperado de: http://www. redalyc.org/pdf/140/14032016007.pdf

González Apodaca, E. \& Rojas, A. (2013) Proyectos locales, autonomía educativa y resistencia indígena. En: Bertely, M., Dietz, G. \& Díaz Tepepa, G. (coords.), Multiculturalismo y Educación 2002-2011. Estados de conocimiento (pp. 383413). México: ANUIES/COMIE.

Guerrero, A. \& Massieu, Y. (2012). Maíz transgénico, milpa y comunalidad: una mirada desde la Sierra Juárez de Oaxaca. Veredas. Especial, 239-260. México: UAM-Xochimilco. Recuperado de: http://148.206.107.15/biblioteca_digital/articulos/12-648-9374nsp.pdf

Gutiérrez Aguilar, R. (2015). Horizonte ComunitarioPopular. Antagonismo y producción de lo común en América Latina. Puebla: BUAP.

Haesbaert, R. (2013). Del mito de la desterritorialización a la multiterritorialidad. Cultura y representaciones sociales, 8(15), 9-41.

IEEPO/ESCI (2007). Secundaria para la atención de comunidades pertenecientes a los pueblos originarios de Oaxaca. (Versión sintética). Oaxaca: IEEPO/ESCI.

INEGI (2015). Encuesta Intercensal (2015). Panorama sociodemográfico de México 2015. México: INEGI.Recuperadode:http://www3.inegi.org. $\mathrm{mx} /$ sistemas/biblioteca/ficha.aspx? upc $=70$ 2825078065

Jiménez Naranjo, Y. (2009). Cultura comunitaria y escuela intercultural. México: CGEIB/SEP.

Lave, J. \& Wenger, E. (1991). Situated Learning. Legitimate peripheral participation. New York: Cambridge University Press.

López Aguilar, M. (2013). Una reforma “educativa” contra los maestros y el derecho a la educación. El Cotidiano (179) 55-76. Recuperado de: www. elcotidianoenlinea.com.mx/pdf/17905.pdf

Olmeda, Juan C. (2014). El péndulo descentralizaciónrecentralización y su aplicación a la reforma educativa en México. Relaciones Internacionales, Estrategia y Seguridad, 9(2), 143-164. Recuperado de: http://www.scielo.org.co/ scielo.php?script=sci_arttext\&pid=S190930632014000200007\&lng=en\&tlng=es 
Maldonado, B. (2002). Los indios en las aulas: Dinámica de dominación y resistencia en Oaxaca. México: CONACULTA/INAH.

Mercado, R. (1991). Los saberes docentes en el trabajo cotidiano de los maestros. Infancia $y$ Aprendizaje, 55, 59-72.

Meyer, L. (2010). Hacia una alternativa de formación indígena bilingüe e intercultural: una pedagogía liberadora desde la "comunalidad". Revista Latinoamericana de Inclusión Educativa, 4(1) 83-103 http://www.rinace.net/rlei/numeros/ vol4-num1/art4.pdf.

Meyer, L. \& Soberanes, F. (2009). El nido de lengua. Orientación para sus guías. México: CNEII/ CMPIO/CSEIIO.

Rendón Monzón, J.J. (ed.) (2003). La comunalidad. Modo de vida en los pueblos indios. México: CONACULTA, pp. 91-108.

Rengifo, G. (2009). Las chacras de la escuela y de la niñez, y la descolonización de la educación. Ponencia. Congreso Internacional de Educación: Bodas de Oro. Urubamba, Cusco: La Salle. Recuperado de: http://www.pratecnet.org/pdfs/LaSalle50.pdf
Rivera Cusicanqui, S. (2008). Gestión pública intercultural. Pueblos originarios y Estado. La Paz: SNAP/Azul Editores.

Rockwell, E. (2011). La experiencia etnográfica. Buenos Aires: Editorial Paidós.

Santos, B. (2007). Beyond Abyssal Thinking: From Global Lines to Ecologies of Knowledges. Review. Recuperado de: http://www.boaventuradesousasantos.pt/media/Beyond Abyssal_Thinking_Review_2007.pdf

Stavenhagen, R. (2010). Los pueblos originarios: el debate necesario. Buenos Aires: CTA Ediciones/CLACSO. Recuperado de: http:// bibliotecavirtual.clacso.org.ar/clacso/coediciones/20101117084419/Stavenhagen.pdf

Vanhulst, J. y Beling, A. (2013). Buen vivir: la irrupción de América Latina en el campo gravitacional del desarrollo sostenible. Revista de La Red Iberoamericana de Economía Ecológica. 21, 1-14. Recuperado de: http://www.redibec. org/IVO/rev21_01.pdf

Velasco, H. \& Díaz de Rada, A. (1997). La lógica de la investigación etnográfica. Un modelo de trabajo para etnógrafos de escuela. Madrid: Editorial Trotta. 